\title{
Anabases
}

ANABASES Traditions et réceptions de l'Antiquité

$10 \mid 2009$

Varia

\section{Les marbres antiques retrouvent des couleurs : apport des recherches récentes et débats en cours}

Adeline Grand-Clément

\section{(2) OpenEdition}

1 Journals

Édition électronique

URL : http://journals.openedition.org/anabases/721

DOI : 10.4000/anabases.721

ISSN : 2256-9421

Éditeur

E.R.A.S.M.E.

Édition imprimée

Date de publication : 1 octobre 2009

Pagination : 243-250

ISSN : 1774-4296

\section{Référence électronique}

Adeline Grand-Clément, « Les marbres antiques retrouvent des couleurs : apport des recherches

récentes et débats en cours », Anabases [En ligne], 10 | 2009, mis en ligne le 01 octobre 2012, consulté le 20 octobre 2019. URL : http://journals.openedition.org/anabases/721 ; DOI : 10.4000/anabases. 721

(c) Anabases 


\section{Les marbres antiques retrouvent des couleurs: apport des recherches récentes et débats en cours}

Adeline Grand-Clément

La POLYCHROMIE DE L'ART GREC n'est pas une découverte récente. Dès le début du XIX siècle, les savants européens ont dû se rendre à l'évidence: les Hellènes avaient l'habitude de peindre leurs sculptures et leurs édifices, y compris ceux de marbre blanc. C'est le temps, parfois secondé par la main de l'homme, qui a estompé ou effacé la vivacité des coloris antiques, biaisant la vision que nous avons aujourd'hui des vestiges du passé. Mais, une fois le principe admis, est-il possible de donner à voir la polychromie des Anciens? Peut-on restituer aux ouvres exposées dans les musées leurs couleurs d'antan, afin de recréer l'univers chromatique dans lequel évoluaient les Grecset les Romains? Et, au-delà même de la question de la «faisabilité", doit-on tenter de le faire? En somme, faut-il repeindre les marbres antiques - ou les copies en plâtre des marbres antiques? De telles interrogations mettent en jeu notre rapport à l'Antique et la valeur patrimoniale que nous attribuons aux monuments et aux œuvres d'art dits «classiques». Elles invitent aussi à réfléchir aux conditions de diffusion et de valorisation de la recherche scientifique au sein de l'ensemble de la société.

Les premières tentatives de reconstitution en couleurs voient le jour dès le XIX ${ }^{\mathrm{e}}$ siècle, d'abord pour les édifices, et sous une forme graphique. En 1851, dans les planches chromolithographiées de L'architecture polychrome chez les Grecs, Jacques-Ignace Hittorff propose un "état restauré» de la polychromie du temple B de l'acropole de Sélinonte ${ }^{1}$. Son travail inspire les jeunes architectes pensionnaires de l'Académie de France à Rome, qui dessinent, dans leurs envois, des édifices bariolés, à partir des vesti-

1 J.-I. HitToRfF, Restitution du temple d'Empédocle à Sélinonte. L'architecture polychrome chez les Grecs, Paris, Firmin Didot, 1851, pl. I à VI. 
ges qu'ils ont étudiés et relevés sur les sites d'Italie et de Grèce ${ }^{2}$. Certains se lancent aussi dans des reconstitutions tridimensionnelles. Les Allemands jouent un rôle pionnier: en 1816, les sculptures du fronton du temple d'Aphaia à Égine, récemment découvertes, sont exposées dans la Glyptothèque de Munich devant une copie en plâtre vivement colorée de la façade de l'édifice, exécutée par Leo von Klenze ${ }^{3}$. En France, Hittorff réalise un temple grec miniature, entièrement polychrome, à la demande du prince Jérôme Napoléon; la maquette est exposée à Paris, au salon de $1859^{4}$.

De telles expérimentations existent également pour les statues, mais apparaissent plus tardivement. Les fouilles de l'Acropole d'Athènes livrent à la fin du XIX ${ }^{\mathrm{e}}$ siècle la riche série des korai, dont la polychromie a été préservée en raison de leur enfouissement peu après les destructions de la seconde guerre médique. Ces statues de jeunes filles sont alors immortalisées dans leur parure chatoyante par les aquarelles du peintre suisse Louis Émile Gilliéron. C'est ce précieux relevé graphique qui permet ensuite à son fils de peindre les moulages en plâtre de cinq de ces korai, entre 1911 et 1924, pour restituer l'apparence qu'elles avaient au moment de leur découverte. Le cap décisif de la remise en couleurs intégrale n'est pas encore franchi. C'est plutôt en Allemagne, à l'initiative de Georg Treu, qui dirige le musée de Dresde à partir de 1882, que les premières expérimentations sont réalisées. L'archéologue encourage en effet des artistes contemporains à peindre des copies en plâtre d'œuvres classiques, afin de recréer l'aspect supposé d'origine ${ }^{5}$. L'entreprise fait quelques émules: en 1891, Edward Robinson décide d'exposer deux moulages repeints de statues antiques en marbre, au musée des Fine Arts à Boston, dont il est le directeur ${ }^{6}$. Cependant, les méthodes d'analyse restent rudimentaires et ne permettent pas de mener à bien ces entreprises avec toute la rigueur scientifique nécessaire. Discréditées auprès de la communauté savante, perçues comme des fantaisies d'artistes, elles se raréfient au $\mathrm{Xx}^{\mathrm{e}}$ siècle.

2 M.-F. Billot, "Recherches aux XVIII et XIX ${ }^{\mathrm{e}}$ siècles sur la polychromie de l'architecture grecque", Paris-Rome-Athènes, le Voyage en Grèce des architectes français auX XIX et XX siècles, Paris, École nationale des Beaux-Arts, 1982, p.61-125.

3 La façade en plâtre peint reste en place jusqu'à la fin du XIX ${ }^{\mathrm{e}}$ siècle. L'archéologue Adolf Furtwängler, devenu directeur de la Glyptothèque, décide alors d'exposer dans le musée une nouvelle reconstitution, plus fiable, étayée sur les fouilles qu'il a lui-même menées à Égine.

4 La maquette a été détruite lors de la Commune, mais une reconstitution a été réalisée et présentée aux musées de Montauban et d'Arles à l'occasion de l'exposition «Ingres et l'Antique»: voir P. PiCARD-Cajan (dir.), Ingres et l'Antique. L'illusion grecque, Arles, Actes Sud, 2006, p. 193-195.

5 A. BLÜHM, "Living Colour. A short story of colour on sculpture in the $19^{\text {th }}$ century", The Colour of Sculpture, 1840-1910, Amsterdam-Leeds, 1996, p. 11-60.

6 Il s'agit de l'Hermès de Praxitèle, qui vient d'être découvert à Olympie par les Allemands, et d'une statue de Vénus Genitrix. Voir le texte apologétique d'E. Robinson, The Hermes of Praxiteles and the Venus Genitrix, Boston, A. Mudge and son, 1892. 
Depuis une dizaine d'années, cependant, on voit renaittre les projets de reconstitutions tridimensionnelles de la polychromie antique, plus audacieuses et suggestives que les traditionnelles reconstitutions graphiques. La couleur est en effet à l'honneur ${ }^{7}$. Les missions de conservation et de restauration dévolues aux musées européens ont contribué à susciter ces projets; le développement de nouveaux moyens technologiques d'investigation les ont rendus possibles. Différents outils permettent désormais de relever, de cartographier et d'analyser avec un degré de précision et de fiabilité remarquable les vestiges de couleur encore présents sur la surface des objets ${ }^{8}$. Un colloque international, organisé par Philippe Jockey, vient de se tenir à l'École française d'Athènes sur «Les arts de la couleur en Grèce ancienne... et ailleurs» (23-25 avril 2009) ${ }^{9}$. Il a permis de faire le point sur l'état actuel des recherches et des résultats obtenus. Les communications et les discussions ont donné l'occasion de confronter les approches et les méthodes employées en Europe.

Dans l'une des communications du colloque, «The so-called "Alexander Sarcophagus", painting Materials and Techniques", Ulrich Koch-Brinkmann a présenté le fruit des recherches qu'elle effectue à Munich aux côtés de l'archéologue allemand Vinzenz Brinkmann (conservateur du département des Antiquités du Liebieghaus, Francfort). L'intervention concernait plus spécifiquement l'étude de la polychromie originelle du sarcophage dit "d'Alexandre» provenant de Sidon et conservé au musée d'Istanbul. Cette pièce en marbre, datée d'environ 320 avant notre ère, a en effet conservé d'importants vestiges de couleurs, encore observables à l'œil nu. Son décor en bas-relief

7 Parmi les nombreuses publications récentes consacrées à la couleur dans l'art antique, citons M.A. Tiverios et D.S. Tsiafakis, Color in Ancient Greece. The Role of Color in Ancient Greek Art and Architecture 700-31 B. C., Thessalonique, Aristotle University of Thessaloniki, Lambrakis Research Foundation, 2002; L. Cleland et K. STEARS, Colour in the Ancient Mediterranean World, Oxford, BAR, 2004; H. BrÉCOULAKI, La peinture funéraire de Macédoine: emplois et fonctions de la couleur, $I V^{e}-I I^{e}$ s. av. J.-C., Paris, De Boccard, 2006; S. Descamps-Lequime (dir.), Peinture et couleur dans le monde grec antique, Paris, Musée du Louvre, 2007.

8 Deux types d'analyses se complètent: celles dites «de surface» (détection des traces de couleur: examen à la loupe binoculaire et au vidéo-microscope, spectrométrie de fluorescence $\mathrm{X}$ et infrarouge, photographie sous rayons UV, lumière rasante), celles dites «structurelles» (identification des pigments; on privilégie aujourd'hui les méthodes dites «non destructives", qui nécessitent des prélèvements infimes). Sur toutes ces méthodes, voir B. Bourgeois et Ph. JOCKey, "Approches nouvelles de la polychromie des sculptures hellénistiques de Délos”, CR de l'AIBL 2001, p. 629-665.

9 On ne peut manquer de souligner la qualité et la richesse des communications qui y ont été présentées; nous ne pourrons malheureusement les évoquer toutes ici, loin s'en faut. Elles témoignent du dynamisme de la recherche en matière de polychromie, sur des medias très variés: peintures minoennes et mycéniennes, terres cuites hellénistiques, textiles, tombes de la "Vallée des rois thraces», peintures funéraires d'Étrurie et d'Italie du sud, sculpture perse achéménide... Les aspects anthropologiques de la question ont également été abordés. 
était à l'origine entièrement recouvert de peinture. Les analyses ont permis d'identifier la présence d'une vingtaine de pigments différents, appliqués de façon à produire des effets d'ombre et de lumière, rehaussant ainsi l'effet plastique et le volume des figures sculptées. L'étude du sarcophage de Sidon ne constitue nullement une entreprise isolée: elle s'inscrit dans le cadre d'un programme initié au début des années 1980 au DoernerInstitut de Munich. Les méthodes d'investigation mises au point par V. von Grave ont permis de traquer les vestiges de couleurs sur les œuvres en marbre de la Glyptothèque de Munich, en particulier les sculptures du temple d'Aphaia à Égine. Vinzenz Brinkmann a poursuivi les analyses sur des pièces conservées ailleurs en Europe, notamment la frise du trésor de Siphnos, exposée à Delphes ${ }^{10}$.

L'initiative allemande menée avec l'appui technique du Doerner-Institut de Munich a conduit à la mise en place d'une collaboration avec deux autres équipes, l'une italienne, l'autre danoise. La première a travaillé de 1998 à 2003 à l'étude de la polychromie de la célèbre statue d'Auguste dite de Prima Porta, conservée au Musée du Vatican. Paolo Liverani, de l'Université de Florence, a eu en charge la direction du projet. Il était également présent lors du colloque d'Athènes; son intervention, intitulée "Reflections about the color coding in Roman art", abordait la question, plus large, de la signification sociale des couleurs du vêtement dans le monde romain. L'équipe danoise, elle, s'est consacrée à l'étude du portrait de Caligula conservé à la Glyptothèque Ny Carlsberg de Copenhague. Jan Stubbe Østergaard, qui supervisait l'opération, a détaillé lors du colloque d'Athènes le vaste programme de recherche entrepris depuis par le CPN, le Copenhaguen Polychromy Network (son intervention s'intitulait «Research on ancient sculptural polychromy in the Ny Carlsberg Glyptotek, Copenhaguen: introduction and first results»). Le projet mobilise des spécialistes de différentes disciplines autour d'une campagne de détection des vestiges de couleur sur une sélection de marbres conservés au musée. Les pièces sont étudiées in situ, sous le regard curieux et étonné des visiteurs. Une campagne d'information menée en parallèle permet d'expliquer au public la nature des recherches effectuées et de le sensibiliser à la question de la polychromie antique. Le retour est excellent: les visiteurs font preuve d'intérêt pour ces avancées scientifiques qui remettent en cause leur vision traditionnelle de l'art classique.

C'est précisément le souci de rendre accessibles les progrès de la recherche au grand public qui a conduit les trois équipes à s'essayer à des reconstitutions en trois dimensions, grandeur nature, destinées à être exposées dans des musées. Elles ont ainsi renoué les fils d'une tradition née au XIX ${ }^{\mathrm{e}}$ siècle, mais abandonnée au $\mathrm{XX}^{\mathrm{e}}$ siècle - même si, dans les années 1970, quelques essais isolés avaient vu le jour, comme pour la Korè au

10 Sur les résultats de ses investigations, reposant principalement sur l'observation des vestiges sous lumière rasante, voir V. BRINKMANN, Die Polychromie der archaischen und frühklassischen Skulptur, Munich, Biering und Brinkmann, 2003, et, plus spécifiquement, sur la frise de Siphnos, V. BRInKmann, Beobachtungen zum formalen Aufbau und zum Sinngehalt der Friese des Siphnierschatzhauses, Ennepetal, Biering und Brinkmann, 1994. 
peplos de la galerie des moulages de l'Institut d'Archéologie classique de l'Université de Cambridge ${ }^{11}$. L'entreprise conduite autour de Vinzenz Brinkmann était de plus grande envergure. Il s'agissait de faire prendre conscience au public du rôle joué par les couleurs dans la signification des sculptures antiques, et de mettre à l'épreuve les informations récoltées jusque-là, de passer de la théorie à la pratique: dans quelle mesure la polychromie contribuait-elle à modifier l'apparence des œuvres? L'objectif était donc double, à la fois pédagogique et expérimental. L'exécution des différentes étapes du processus de mise en couleurs devait permettre de confirmer ou d'infirmer les hypothèses, de retrouver et de comprendre les gestes des artisans antiques. Une vingtaine de sculptures en marbre a ainsi été sélectionnée. Les copies (principalement des moulages en plâtre) ont été peintes en tenant compte des analyses effectuées. L'une des premières pièces ainsi réalisées a été l'archer du fronton ouest du temple d'Aphaia à Égine, dont le collant et le vêtement bigarrés ont servi à lancer l'exposition, intitulée Bunte Götter, "Les dieux bariolés». Elle a d'abord été présentée à la Glyptothèque de Munich, en 2003, et proposait au visiteur d'admirer les originaux à côté des copies peintes ${ }^{12}$. Il y avait parmi elles la réplique en plâtre de l'Auguste de Prima Porta et celle, en marbre synthétique, du portrait de Caligula. L'exposition a ensuite fait le tour de l'Europe, s'enrichissant parfois de nouvelles reconstitutions ${ }^{13}$. Elle a été accueillie au musée du Vatican et à la Glyptothèque de Copenhague, les deux autres musées partenaires du projet, puis à Bâle, Athènes, Amsterdam, Hambourg, Istanbul, pour finalement traverser l'Atlantique, jusqu'à Harvard ${ }^{14}$. Une sélection de pièces a également été intégrée à une exposition organisée en 2008 par le J. Paul Getty Museum (Los Angeles) sur la place de la polychromie dans l'art occidental, depuis l'Antiquité: The Color of Life ${ }^{15}$.

11 Un moulage en plâtre de la statue a été peint à l'initiative de Robert Cook, en 1975. Il a dû faire l'objet d'une restauration en 1996, car la vivacité des couleurs s'estompait. Sur cette reconstitution, voir http://www.classics.cam.ac.uk/museum/peplos_kore/ (site consulté le 8 juin 2009). L'équipe de Cambridge a également réalisé, plus récemment, une copie polychrome de la dame d'Auxerre: voir http://commons.wikimedia.org/wiki/ File:Lady_of_Auxerre_University_of_Cambridge.jpg/(site consulté le 8 juin 2009). Le catalogue de l'exposition fournit une documentation iconographique riche et abondante: V. Brinkmann, R. WünsChe, U. Wurnig (dir.), Bunte Götter, die Farbigkeit antiker Skulptur, Munich, Staatliche Antikensammlungen und Glyptothek, 2004.

13 Par exemple pour la Korè au peplos, il existe désormais deux copies peintes, l'une qui la figure sous les traits d'Athéna, l'autre d'Artémis; de même, on dispose à ce jour de deux versions du portrait de Caligula, conservées l'une à Munich, l'autre à Copenhague.

14 Deux nouvelles versions du catalogue ont ainsi été publiées: A. Gramiccia (dir.), I colori del bianco. Policromia nella scultura antica, Rome, De Luca, 2004; A. Brauer et S. EbBinghaus, Gods in Color: painted sculpture of classical antiquity, Munich, Stiftung, 2007.

15 Le catalogue vient de paraître: R. PANZANelli (ed.), The Color of Life: polychromy in sculpture from antiquity to the present, Los Angeles, The J. Paul Getty Museum, 2008. Un dossier de présentation est accessible en ligne: http://www.getty.edu/art/exhibitions/ color_of_life/ (site consulté le 8 juin 2009). 
Cette série d'expositions, qui n'a pas encore touché la France, a reçu un accueil assez enthousiaste de la part du public, une fois surmonté le sentiment de répulsion que suscite souvent, au premier regard, la vision de ces sculptures bariolées. La réaction a été plus critique au sein du milieu scientifique, souvent sceptique quant au degré de fiabilité des reconstitutions polychromes. Dans quelle mesure l'analyse des pigments permet-elle de garantir la fiabilité des repeints? Cette analyse a-t-elle été conduite en faisant appel à l'ensemble des moyens technologiques de détection et d'identification dont disposent les chercheurs aujourd'hui? Le mode d'application, par aplats totalement couvrants, correspond-il réellement à celui employé par les artisans grecs et romains? La peinture masquait-elle autant la surface du marbre? Quid du traitement qui donnait aux statues leur lustre final, de cette ganôsis dont on sait encore fort peu de choses? Jusqu'où aller dans la reconstitution? Ne faut-il pas laisser vierge de couleur les parties sur lesquelles on n'a pu détecter aucune trace de pigment - parti-pris adopté pour l'Auguste de Prima Porta - plutôt que de choisir de façon arbitraire tel ou tel pigment? Le recours à du plâtre, en lieu et place du marbre, ne fausse-t-il pas l'effet original de la couche de peinture sur la surface cristalline de la pierre? Les interventions et remarques d'Ulrich KochBrinkmann, de Paolo Liverani et de Jan Stubbe Østergaard, lors du colloque d'Athènes, montrent qu'ils ont pleinement conscience des limites de ce type d'expérimentation et mesurent le caractère imparfait et perfectible de leurs reconstitutions tridimensionnelles. Celles-ci restent nécessairement hypothétiques et sujettes à discussion, mais elles offrent toutefois l'intérêt d'ébranler, au sein des sociétés occidentales, la vision canonique d'une Antiquité drapée de blanc. Les copies peintes ne sont pas conçues comme une fin en soi, le fruit de recherches abouties, mais bien comme le moyen de conduire ces recherches, un instrument au service de l'enquête archéologique, susceptible de faire surgir de nouveaux problèmes et d'alimenter ainsi la réflexion.

Une équipe française, également intéressée par la question de la polychromie antique, a procédé à d'autres choix, qui indiquent l'existence d'une alternative. L'organisateur du colloque d'Athènes, Philippe Jockey, de l'Université d'Aix-en-Provence, a effectué en 1993 une première campagne d'analyse des marbres hellénistiques de l'île de Délos, l'un des sites fouillés par l'École française d'Athènes, en utilisant la photographie sous rayons ultra-violets. En 1999, il a alors entrepris, avec Brigitte Bourgeois, du Centre de Recherche et de Restauration des Musées de France, un vaste programme de recherche. Un balayage au vidéo-microscope des collections du musée a révélé l'étendue de la polychromie des marbres et mis en évidence la présence de feuilles d'or, appliquées en complément de la peinture. Une seconde étape a consisté à identifier la nature des pigments utilisés par les artisans déliens ${ }^{16}$. Le colloque d'Athènes a été l'occasion

16 Citons, parmi les publications présentant la nature des analyses effectuées et les premiers résultats obtenus: B. Bourgeois et Ph. Jockey, "Le geste et la couleur. Stratégies de mise en couleurs de la sculpture hellénistique de Délos", Bulletin archéologique du Comité des Travaux Historiques et Scientifiques 30 (2003), p.65-77; IDEM, "Le marbre, l'or et la couleur. Nouveaux regards sur la polychromie de la sculpture hellénistique de Délos", dans 
de présenter une synthèse des résultats obtenus. Dans sa communication «L'or et les couleurs de la sculpture hellénistique de Délos: ombres et lumières», Brigitte Bourgeois a ainsi exposé la composition de la palette délienne. Elle a également souligné la virtuosité des artisans grecs dans la modulation des teintes et les jeux d'ombre et de lumière. Si des questions demeurent encore en suspens, les résultats de l'enquête invitent à réévaluer le rôle de la polychromie des marbres grecs, en s'interrogeant notamment sur les conditions matérielles de leur présentation et de leur réception, chez les Anciens.

Comment, dès lors, rendre compte des indices patiemment amassés? Philippe Jockey et Brigitte Bourgeois ont opté pour un mode de présentation différent de celui retenu par l'équipe allemande. Point de copie en plâtre ni de pinceau, mais un clavier et un écran d'ordinateur: ils ont préféré recourir à l'imagerie numérique. Le travail de saisie informatique est long et fastidieux, mais, une fois tous les paramètres enregistrés, il offre des potentialités considérables. En effet, les reconstitutions tridimensionnelles virtuelles peuvent aisément être modifiées, amendées, rectifiées ou complétées au gré des avancées de la recherche. Le programme de numérisation et de restitution chromatique des statues déliennes a nécessité la collaboration d'une architecte, Fabricia Fauquet. Celle-ci a expliqué le sens de la démarche lors du colloque d'Athènes ("L'illusion des couleurs: des réalités antiques à l'imagerie numérique contemporaine»). Mené à bien entre 2006 et 2008, en partenariat avec l'Institut des sciences et technologie de l'information de Pise, le projet a concerné cinq statues: une Aphrodite et un Apollon, le Gaulois blessé, l'Artémis au cerf et la copie du Diadumène. Fabricia Fauquet a fait la démonstration, sur l'écran d'un ordinateur, de l'intérêt de l'outil ainsi créé. Grâce aux données enregistrées, nous pouvons désormais disposer de reconstitutions virtuelles de ces œuvres, en trois dimensions, les examiner sous tous les angles, et faire varier leur polychromie en fonction des paramètres que l'on introduit dans le programme. Cela permet de visualiser et de comparer différentes hypothèses concernant la cartographie et la coloration des surfaces peintes. L'intérêt de l'outil informatique, qui sera sans doute diffusé sous la forme d'un DVD, afin d'en faire profiter un large public, va même au-delà. Il offre la possibilité de re-contextualiser l'œuvre en simulant les conditions dans lesquelles elle était exposée au regard des Déliens. On sort ainsi du cadre du musée ou de la vitrine d'exposition, pour replacer le marbre dans son environnement d'origine. Nous avons ainsi une idée de la façon dont la polychromie pouvait être mise en scène et perçue. Fabricia Fauquet a présenté le cas de l'Artémis au cerf, dont le lieu de découverte est bien connu. La reconstitution virtuelle replace la sculpture polychrome dans le fond de la cour de la maison où elle a été retrouvée. En simulant la course du soleil on peut avoir un aperçu du spectacle qu'elle offrait au visiteur délien à différents moments de la journée. Reléguée dans l'ombre le matin et le soir, c'est en début d'aprèsmidi qu'elle étincelait de couleurs.

S. Descamps-Lequime (dir.), Peinture et couleur dans le monde grec antique, Paris, Musée du Louvre, 2007, p. 163-191. 
Parce qu'elle nous permet d'accéder à une perception globale de l'œuvre dans son environnement physique, et de redonner ainsi vie aux marbres antiques, l'imagerie virtuelle constitue à n'en pas douter un outil précieux pour les recherches futures ${ }^{17}$. Dès lors, faut-il choisir entre la méthode allemande et la méthode française? Assurément, elles ne sont pas exclusives et contribuent chacune à leur façon à changer notre regard sur le goût des Anciens en matière de couleurs. Il est illusoire de prétendre refabriquer de l'Antique, et vain de vouloir le faire. En revanche, il est possible de réfléchir à la façon dont nous avons fait des productions gréco-romaines les emblèmes d'un "art classique", en les mettant dans les vitrines de nos musées. Et c'est en oubliant les leçons d'Hegel et de Winckelmann que nous accepterons enfin l'intrusion de la couleur sur la surface du marbre antique. Gageons que la publication prochaine des Actes du colloque d'Athènes, prévue dans la collection des "Suppléments» au Bulletin de Correspondance Hellénique, nous y aidera.

Adeline Grand-Clément

Université de Toulouse (UTM)
PLH (ERASME)
Pavillon de la Recherche
5, allées Antonio-Machado
F-31058 Toulouse Cedex 9
adelinegc@yahoo.fr

17 L'outil informatique n'est performant que lorsque le chercheur l'utilise à bon escient, en disposant de données solides et fiables. Les reconstitutions polychromes de la Virtual Sculpture Gallery proposées en ligne par un groupe de l'Université de Malibu sont d'un tout autre ordre (http://mandarb.net/virtual_gallery/sculptures.shtml/ site consulté le 8 juin 2009). Il s'agit de simples photographies d'œuvres antiques scannées et retouchées assez librement grâce au logiciel Photoshop, à partir des vestiges de couleur observables à l'œil nu. Elles ont néanmoins le mérite de sensibiliser les étudiants en art classique à la question de la polychromie. 